\title{
LA PLACE DES ÉCRIVAINES AUTOCHTONES DANS LA LITTÉRATURE MONDIALE : LE CAS DU BRÉSIL ET DU QUÉBEC ${ }^{1}$
}

\author{
Rita Olivieri-Godet ${ }^{2}$
}

\begin{abstract}
Résumé: Les créations littéraires et artistiques autochtones actuelles au Brésil et au Québec sont traversées par l'émergence de voix de femmes dont les œuvres portent leur subjectivité féminine et un désir d'émancipation sociale et littéraire. Militantes et écrivaines, ces femmes essayent de se frayer un chemin au sein de nos sociétés occidentales par le biais de leur écriture, considérée comme un lieu utopique de survie et de résistance, et de leurs actions qui se reflètent dans leurs trajectoires organisationnelles. En s'inscrivant dans un processus contemporain de transculturalité, la littérature amérindienne apparaît comme un lieu utopique de survie et de résistance, mais aussi de médiation qui fait éclore des formes originales d'expression artistique, sans renoncer à une réappropriation mémorielle du territoire géoculturel des ancêtres. En analysant un corpus émergent et peu étudié (francophone et lusophone), nous chercherons à dégager les singularités de ces voix féminines qui fondent de nouveaux paradigmes et contribuent ainsi à la reconfiguration des systèmes littéraires et des imaginaires. Elles mettent en scène des processus d'articulation des espaces qui permettent la circulation et la (re)contextualisation d'expériences et de cultures singulières.
\end{abstract}

Mots-clés: Littérature autochtone contemporaine; Ecrivaines autochtones; transculturalité ; Brésil ; Québec

\section{O LUGAR DAS ESCRITORAS INDÍGENAS NA LITERATURA GLOBAL: O CASO DO BRASIL E QUEBEC}

Resumo: Nas criações literárias e artísticas indígenas no Brasil e no Quebec atuais, manifestam-se vozes de mulheres cujas obras expressam a subjetividade feminina e um desejo de emancipação social e literária. Militantes e escritoras, essas mulheres constroem caminhos no seio das sociedades ocidentais através de sua escrita e de suas ações que se refletem nas suas trajetórias organizacionais. Inserindo-se em um processo contemporâneo de transculturalidade, a literatura indígena revela-se como um lugar utópico de

\footnotetext{
${ }^{1}$ Cet article reprend des éléments développés dans notre ouvrage Ecrire l'espace des Amériques: représentations littéraires et voix de femmes amérindiennes, Peter Lang, 2019.

2 Professora titular de literatura brasileira da Université Rennes 2-França. Doutora em Teoria literária e literatura comparada pela USP, com pós-doutorado em literatura comparada na Université Paris 10 Membro da equipe ERIMIT-Equipe de Recherches Interlangues "Mémoires, Territoires et Identités". Endereço eletrônico: ritagodet20@gmail.com
} 
sobrevivência e de resistência, mas também de mediação, que propicia formas originais de expressão artística, mas que, no entanto, não renuncia a uma reapropriação memorial do território geocultural dos ancestrais. Procurarei detectar, em um corpus emergente e pouco estudado (francófono e lusófono), as singularidades dessas vozes femininas que fundam novos paradigmas e contribuem para a reconfiguração dos sistemas literários e dos imaginários. Destacarei o fato de que as escritoras indígenas encenam processos de articulação dos espaços que permitem a circulação e a (re)contextualização de experiências e de culturas singulares.

Palavras-chave: Literatura indígena contemporânea; Escritoras indígenas; transculturalidade; Brasil ; Québec

Nous partons du constat que les créations littéraires et artistiques autochtones actuelles au Brésil et au Québec sont traversées par l'émergence de voix de femmes dont les œuvres portent leur subjectivité féminine et un désir d'émancipation sociale et littéraire. En s'inscrivant dans un processus contemporain de transculturalité, la littérature amérindienne apparaît comme un lieu utopique de survie et de résistance, mais aussi de médiation qui fait éclore des formes originales d'expression artistique, sans renoncer à une réappropriation mémorielle du territoire géoculturel des ancêtres. En analysant un corpus émergent et peu étudié (francophone et lusophone), nous chercherons à dégager les singularités de ces voix féminines qui fondent de nouveaux paradigmes et contribuent ainsi à la reconfiguration des systèmes littéraires et des imaginaires.

Ce qui interpelle lorsqu'on examine la prise de parole des femmes autochtones, c'est leur position d'avant-garde au sein de cette production, depuis les années 1970 -lorsque An Antane Kapesh, au Québec, et Eliane Potiguara, au Brésil, produisent leurs premiers textes-ainsi que le rôle absolument fondamental de l'écriture littéraire dans leur processus de reconstruction identitaire en tant qu'acteurs sociaux.

L'émergence des voix autochtones dans les lettres brésiliennes et québécoises contribue à la reconfiguration de l'imaginaire sur les Amérindiens, tout en ouvrant un dialogue avec la tradition littéraire et les intertextes sociaux. La diversité des Peuples Premiers et celle de leurs productions intellectuelles sont en rapport avec les différentes phases 
d'interlocution avec les sociétés nationales : récits oraux traditionnels, textes ethnographiques, récits mythiques, essais politiques, " auto-anthropologie ", littérature de jeunesse, textes romanesques et poétiques, production audiovisuelle et musicale. Le passage de l'oral à l'écrit et l'appropriation de la langue nationale (portugaise ou française) ont engendré de nouvelles formes et thématiques et ont contribué à élargir l'insertion de ces productions dans l'espace culturel brésilien et québécois. L'examen de ce processus révèle notamment le rôle de l'écriture comme une tactique fondamentale à laquelle les Amérindiens ont recours pour établir un dialogue avec la culture hégémonique et s'affirmer en tant que sujet de leur propre histoire. De sujet de la représentation, l'Amérindien devient le sujet de l'énonciation dans les lettres brésiliennes et québécoises.brésil

Dans le contexte actuel d'intensification des contacts entre les deux sociétés et de prise de conscience politique par les Amérindiens, on observe que la création artistique devient peu à peu une arme efficace pour s'immiscer dans le marché de la production artistique occidentale et faire bouger l'imaginaire sur les peuples et cultures autochtones. La prise de parole de femmes autochtones se situe au premier plan de ce processus, dans les deux pays, mais pas exactement au même niveau. Force est de constater que malgré la répercussion des discours sociaux sur la brûlante question indienne au Brésil, la création littéraire amérindienne écrite en portugais est encore assez discrète, contrastant avec le système littéraire québécois qui présente un corpus conséquent d'ouvrages écrits par des Amérindiens et, en particulier, par des femmes amérindiennes. Nous nous proposons de dégager les potentialités singulières d'une écriture issue de l'expérience de vie de ces auteures, en tant qu'amérindiennes et femmes, acteurs sociaux doublement discriminés.

L'exploration initiale d'un corpus qui n'est pas encore complètement consolidé, étant donné le travail d'investigation nécessaire à son établissement au Brésil, laisse entrevoir la multiplicité de genres littéraires auxquels les écrivaines ont recours (autofiction, autobiographie, témoignage, chronique, roman, poésie, récits mythiques) ainsi que la tendance à dépasser leurs frontières, en explorant le principe de la polytextualité. L'écriture porte les marques des genres traditionnels de la littérature orale 
amérindienne (mythes, légendes, chants) et innove dans la représentation du temps en faisant usage de l'hétérotemporalité. Nous faisons l'hypothèse que le caractère novateur de cette production est dû notamment au fait que ces écrivaines tirent profit de leur situation liminaire : à la fois ancrée dans leur héritage ancestral et ouverte aux formes artistiques de la contemporanéité immédiate.

Les écritures amérindiennes contemporaines, à l'instar des écritures migrantes, sont, elles aussi, mouvantes, car elles s'ouvrent au croisement de cultures cherchant à dépasser la fracture coloniale et s'inscrivent ainsi dans le processus contemporain de transculturalité qui fait éclore de nouvelles formes de perception du réel et d'expression artistique, sans néanmoins renoncer à exposer les souffrances du déracinement imposé par la colonisation. Cette tension entre le désir de dépassement de la ligne de fracture que la mémoire de la dépossession ravive et la quête de relation est une des constantes de cette production. Cela expliquerait peut-être l'ambivalence qui se dégage des œuvres des femmes autochtones urbaines oscillant entre une poétique de la confrontation, laquelle met en avant les références identitaires, et une poétique de la relation qui promeut la traversée des frontières.

Pour nourrir notre réflexion sur l'écriture de femmes autochtones et ses échos dans le domaine du social et du politique, notre approche porte une attention privilégiée aux questions qui ont trait au rapport de l'écriture à la praxis, à la mémoire, à l'espace et au processus de reconstruction de soi.

Un des axes mis en avant dans nos analyses se rapporte aux relations entre écriture, critique sociale et activisme politique. Cette écriturepraxis portée par la parole de femmes amérindiennes qui parlent au nom de leur peuple inaugure la production littéraire autochtone et son insertion dans le système littéraire du Québec et du Brésil. Nous nous attachons à préciser les éléments discursifs d'une écriture envisagée comme un espace politique de résistance et d'auto-reconstruction ontologique et anthropologique qui cherche à entrelacer les " auto-histoires " (l'histoire personnellement vécue) à la violence et aux traumatismes de l'histoire collective des Amérindiens. Il importe de signaler la répercussion des œuvres pionnières de An Antane Kapesh au Québec et de Eliane Potiguara au Brésil, dans l'organisation 
politique du mouvement indien -et plus particulièrement du mouvement des femmes amérindiennes- et sa contribution à leur émancipation. Ces deux femmes inaugurent les voix/voies de la décolonisation.

Dans la publication de 1976, Je suis une maudite sauvagesse, An Antane Kapesh, auteure d'origine innue, assume, pour mieux le déconstruire, le stéréotype négatif des représentations des Amérindiens. L'écriture est pour elle un acte militant et le récit «basé sur son expérience de vie [...] remet en question le processus de déterritorialisation et d'imposition culturelle fait à son peuple, par l'éducation obligatoire et la sédentarisation " (Olivieri-Godet, 2015, p. 45). Écriture de la dénonciation et de la révolte d'où se dégage une éthique fondée sur le combat comme le seul moyen pour atteindre la reconnaissance.

Lors de la publication de son poème " Identidade indígena », en 1975, Eliane Potiguara militait dans l'organisation du mouvement indien au Brésil qui débutait à peine. Le poème est à la fois dénonciation, résilience et combat, mémoire de l'exclusion et parole libératrice. À l'époque, elle était aussi l'auteure de manifestes et de chroniques. Ces textes écrits pendant et après la Dictature Militaire brésilienne sont réunis dans l'ouvrage qu'elle publie en 2004, Metade cara, metade máscara. Activiste et écrivaine, Eliane Potiguara est à l'origine du GRUMIN-Grupo mulher-educação indígena.

Ces deux écrivaines inaugurent par leur écriture-praxis l'insertion de voix des femmes autochtones dans le système littéraire du Québec et du Brésil. Il est intéressant d'observer le phénomène générationnel qui s'installe, aussi bien dans le Nord que dans le Sud du continent américain : les voix d'An Antane Kapesh, Joséphine Bacon, Rita Mestokosho retentiront dans les œuvres de la nouvelle génération représentée entre autres par Naomi Fontaine, Natasha Kanapé Fontaine, Manon Nolin, Marie-Andrée Gil, Melissa Mollen Dupuis qui revendiquent l'héritage d'un message à passer. L'image du bâton à message, titre du premier recueil poétique de la poète innue Joséphine Bacon, est là pour rappeler que chez les femmes écrivaines amérindiennes « la parole est toujours en voyage" (Bacon, 2009, p. 7). Ce devoir de mémoire auquel Eliane Potiguara fait également allusion ("Eu viverei 200, $500 \mathrm{ou}$ 700 anos /E contarei minhas dores para ti»; "Je vivrai 200, 500 ou 700 ans / et je te raconterai mes douleurs », " Identidade indígena ») est aussi 
partagé par Graça Graúna. La nouvelle génération des écrivaines amérindiennes au Brésil reste encore assez discrète par rapport à son homologue québécoise, mais les noms de Márcia Wayna Kabemba, Márcia Nunes Maciel, Lya Minapoty commencent à s'affirmer dans le paysage littéraire.

Une des thématiques maîtresses explorée par les œuvres qui constituent notre corpus fait appel à la transmission de la mémoire ancestrale et au rôle déterminant que les femmes jouent dans ce processus d'actualisation du savoir des peuples autochtones. Nous nous intéressons à la contre-mémoire qui émane de ces textes en scrutant la singularité du mécanisme de transmission de la mémoire familiale. La réappropriation mémorielle des référents culturels amérindiens (la "mémoire culturelle ", inscrite dans le temps de longue durée) met en scène un autre phénomène, celui de la reterritorialisation symbolique que l'écriture cherche à instaurer en s'emparant de la mémoire de son patrimoine ancestral pour créer son propre habitat. Ainsi, la production littéraire de femmes autochtones pose la question: comment se reconstruire culturellement Indien et réactiver sa culture, après des siècles d'oubli ? C'est à cette question que la création littéraire de Graça Graúna et Eliane Potiguara apporte un début de réponse, en relevant le défi de s'emparer de la mémoire du patrimoine indien millénaire pour poursuivre une quête symbolique et ontologique. Dépossédées du territoire géoculturel de leurs ancêtres, ces écrivaines d'origine potiguara, peuple qui habitait le Nordeste du Brésil, réinventent de nouvelles modalités d'habitabilité psychique (Simon Harel, 2005), en reconfigurant, par le biais de la création littéraire, leur relation avec un milieu qu'elles n'ont pas directement expérimenté : « Je n’ai pas mon village / Mon village c'est ma maison spirituelle / Celle que m'ont léguée mes parents et mes grands-parents / Le plus grand héritage indien. / Cette maison spirituelle / C'est là où je vis depuis ma plus tendre enfance ", écrit Eliane Potiguara (Metade cara, metade máscara, 2004, «Je n’ai pas mon village » / " Eu não tenho minha aldeia », p. 130). La réinvention symbolique du lieu se profile comme une façon de résister à l'usurpation des territoires autochtones. 
Un autre aspect fondamental se rapporte à l'intersection entre la mémoire du territoire autochtone et les paysages urbains. Le roman Kuessipan, à toi (2011) de Naomi Fontaine nous intéresse particulièrement dans la mesure où il donne lieu à des représentations de frontières plus fluides, à la mise en scène des espaces de cohabitation et des échanges, même si les tensions et les conflits sont toujours présents. Les temporalités ne sont plus vécues comme antinomiques mais comme simultanées: de nouvelles mœurs pénètrent la vie de la communauté de la réserve de Uashat d'où l'auteure est originaire, en raison des relations avec l'univers urbain contemporain, et coexistent avec des rituels traditionnels qui occupent une place privilégiée dans le texte dans la mesure où ils symbolisent la résistance culturelle du peuple Innu et sa capacité à se ressourcer. Les rapports des Amérindiens à l'espace urbain et aux territoires autochtones constituent une autre perspective d'analyse en tenant compte de la complexité des figurations qui ont trait : au processus d'interaction entre la dimension spatiale et l'être humain; aux pratiques de l'espace et des paysages; au rapport aux changements des paysages et aux mobilités forcées, mis en scène par les narrateurs ou sujets poétiques, selon que ces paysages renvoient à l'espace urbain ou à des territoires culturels traditionnels. Le poème " La cueillette » (Bleuets et abricots de Natasha Kanapé Fontaine, 2016, p. 41) illustre bien cette question en laissant entrevoir la possibilité de transformer l'expérience dysphorique de la ville.

Un angle d'observation complémentaire renvoie au caractère thérapeutique de l'écriture qui pourrait s'expliquer, d'une part, par l'effet cathartique engendré par la dénonciation des violences (dissolution brutale de l'espace habité amérindien depuis l'arrivée des colonisateurs ; migrations forcées ; violences physiques, morales et symboliques), et d'autre part, par la réappropriation de la mémoire et de l'histoire amérindiennes, tout en inscrivant ces peuples dans l'avenir des sociétés nationales, permettant ainsi au sujet de se reconstruire. Chez Graça Graúna, écrivaine d'origine potiguara, c'est par la poésie que le sujet poétique a accès à la vie, à sa nature intime et profonde, à son rapport au monde. C'est par le "Tissage de la parole », titre d'un de ses poèmes, qu'elle se lance dans l'expérience de l'instant, se donnant 
pour tâche de faire vivre la mémoire de la présence effacée des Amérindiens sur le territoire brésilien. La poésie est, pour cette écrivaine, un lieu de survie.

Le caractère cosmologique et mythique de cette littérature focalise également notre attention sur la relation privilégiée au territoire qui s'exprime entre autres par l'archétype féminin qui engendre la vie. De façon récurrente, les écrivaines puisent dans le mythe du renouvellement pour légitimer la parole de la femme amérindienne, en lui attribuant la force primordiale de transformation qui alimente ses actes et son discours. Pouvons-nous parler d'une littérature cosmologique comme le propose Simon Harel (2017) pour certaines de ces œuvres (celles de Natasha Kanapé Fontaine et de Naomi Fontaine notamment) ? La lecture de ces textes invite le lecteur à une expérience sensorielle de l'espace qui se nourrit de la cosmologie des peuples autochtones par la récurrence d'images qui répercutent l'inscription du corps dans l'espace (Rita Mestokosho, Née de la pluie et de la terre, 2014) en tissant des liens entre l'être humain et le cosmos, en rapport avec la conception amérindienne de l'interdépendance de tous les êtres (physiques et spirituels).

Ces images éveillent, par ailleurs, une sensibilité érotique qui marque les incursions langagières de certains récits (L'amant du lac de Virginia Pésémapéo Bordeleau) et poèmes. L'analyse du sens de l'irruption de la parole amoureuse et du symbolisme érotique s'impose. Il nous semble qu'il s'agit d'une stratégie qui vise à dépasser les traumatismes portés par les discours de la dénonciation des préjugés, des violences et de l'exclusion en faisant le pari de la victoire d'Éros sur Thanatos, comme en témoignent les paroles poignantes de Virginia Pésémapéo Bordeleau dans le prologue de son roman: "L'amant du lac nous apprend que nous ne sommes pas que souffrance, que victimes: nous pouvons aussi être plaisir, exultation des corps, des cœurs. Amours " (L'amant du lac, 2012, p. 10). L'émergence de la subjectivité qui se laisse guider par l'affect, par la force d'Éros qui incite « les potentialités du sensible »(Maffesoli, 2012, p. 262), détermine une pensée symbolique partagée par les œuvres de ces femmes écrivaines autochtones.

Qu'elles vivent dans des villes (Eliane Potiguara, Natasha Kanapé Fontaine, Joséphine Bacon, Graça Graúna, Márcia Nunes Maciel, Márcia Wayna Kambeba), dans de petits villages (Rosângela dos Santos Soares, 
Fátima de Oliveira Silva) ou dans des territoires indiens (Rita Mestokosho), ces femmes-écrivaines jouissent d'une condition " amphibienne ", c'est-à-dire de femmes autochtones habitant l'entre-deux, dans une alternance entre le village indien, la réserve et la ville, tirant profit de dynamiques transculturelles pour innover dans leur expression littéraire : "Cette ville est aussi notre village " ("Esta cidade também é nossa aldeia») écrit Márcia Wayna Kambeba. Leur production oscille entre une poétique de confrontation et une poétique de relation en rapport avec leur position d'entre-deux: résilience et médiation composent leur univers littéraire. Comme le rappelle Daniel Sibony, « il n'y a pas, entre les deux, que des abîmes, il y a des tentatives de passage, des espaces frontaliers et précaires, des lieux de vie et d'invivable » (Sibony, 1991, p. 7).

Des femmes amérindiennes, originaires des deux hémisphères de l'Amérique, lèvent leur voix et rapprochent les deux extrémités du continent par leurs créations littéraires. Leur discours poétique ravive la mémoire de leur ancestralité et rappelle notre histoire partagée inscrite dans les marques de la violence du génocide contre les peuples amérindiens, expérience commune à tous les processus de formation des états nationaux du continent. Leurs œuvres fissurent l'imaginaire colonisé sur l'espace des Amériques, en dévoilant l'oubli historique de l'occupation du territoire par les peuples autochtones.

Exposées aux dimensions simultanées de l'expérience de la migration, qu'elle soit vécue ou symbolique, et qui renvoie aussi bien aux pertes qu'au lieu de vigilance que cette expérience favorise (Harel, 2005, p. 215), les voix de femmes autochtones dévoilent un mode spécifique d'être au monde. Le caractère transculturel de cette écriture nous amène à faire l'hypothèse d'un cheminement d'une écriture ethnique vers une écriture hybride, de la résilience à la médiation.

Vers où évoluent les paroles libérées des femmes amérindiennes ? Chacune d'elles porte sa singularité, mais on remarque néanmoins qu'il y a une tendance à dépasser le discours de la victimisation pour investir dans un imaginaire de la reconstruction et du partage. C'est ce que veut nous faire comprendre l'écrivaine innue Marie-Andrée Gil à travers son commentaire sur son dernier ouvrage Chauffer le dehors(2019): "Je trouve ça super 
important de parler de notre héritage, oui, mais je ne suis pas juste ça. J'écris avec mes valeurs, avec un humour qui vient de ma culture, mais je suis aussi une fille de 32 ans qui vit les choses comme la plupart de ses semblables ». Sa poésie intimiste interroge la complexité de la construction identitaire du sujet : « Je m'élançais / incalculable » (Béante, 2012, p. 45). À son tour, une des leaders du mouvement Idle no more, l'écrivaine anishnaabe de langue anglaise, Leanne Betasamosake Simpson, réaffirme sa volonté de contribuer à la transformation politique des Autochtones, en élaborant des récits qui les libèrent de l'éternel rôle de victimes et inaugurent un lieu de feu, d'humour et d'amour ${ }^{3}$.

L'analyse qui découle des voix de ces femmes-territoires pointe vers leur caractère multiple. Ce sont des paroles de dénonciation de la dépossession et à la fois de désir de partage, paroles de résilience et de désir de relation. De sujet de la représentation à sujet de l'énonciation, ces femmes fondent dans leurs œuvres hybrides une poétique autochtone spécifique qui participe à l'élargissement et à la reconfiguration des dimensions matérielle et symbolique des espaces des Amériques.

La condition " amphibienne » des femmes écrivaines pourrait-elle également contribuer à élargir la perspective critique sur les littératures amérindiennes et, d'une façon plus générale, avoir un impact sur la production critique sur des textes littéraires issus des identités dites " minorisées » ? Cette question, comme d'autres qui émergent à la lecture de ce texte, offre des pistes d'analyse aux travaux de recherche qui s'inscrivent dans une perspective décoloniale. La prise de parole des femmes amérindiennes inaugure un nouveau champ de recherche littéraire en voie de structuration.

\footnotetext{
${ }^{3}$ Voir l'article "Leanne Betasamosake Simpson redefines what it means to be Indigenous in the $21^{\text {st }}$ century » : "I wanted to present an honest reality of what it's like to be Indigenous in 2017. I wanted to get at it from a place of grounded strength, to cast off narratives around victimhood and come at it with fire and humour and love. ", https://quillandquire.com/authors/leanne-betasamosake-simpsonredefines-what-it-means-to-be-indigenous-in-the-21st-century/
} 


\section{References}

\section{Auteures amérindiennes et œuvres citées}

ANTHOLOGIE. NÜRNBERG Dorothea et JECUPE, Olívio (org.). Im Flug der Harpyie/No Vôo da Hárpia. Indigene Poesie und Prosa aus dem brasilianischen Regenwald [Anthologie bilingue allemand-portugais]. Vienne: Löcker, 2015. Textes de : CAINGANG, Iracema Forte ; GOMES, Francisca Aurilene ; KEREXU, Fatima ; PEREIRA, Rosilene Fonseca ; POTIGUARA, Eliane ; SOARES, Rosângela dos Santos.

BACON, Joséphine. Uiesh - Quelque part. Montréal : Mémoire d'encrier, 2018.

BACON, Joséphine. Un thé dans la toundra. Nipishapui nete mushuat. Montréal : Mémoire d'Encrier, 2013.

BACON, Joséphine. Bâtons à message.Tshissinuatshitakana. Montréal : Mémoire d'Encrier, 2009.

BORDELEAU, Virginia Pésémapéo. Ourse bleue. Montréal : Editions de la Pleine Lune, 2007.

BORDELEAU, Virginia Pésémapéo. L'amant du lac. Montréal : Mémoire d'Encrier, 2013.

FONTAINE, Naomi. Manikanetish, Petite Marguerite. Montréal : Mémoire d'Encrier, 2017.

FONTAINE, Naomi. Kuessipan, à toi. Montréal : Montréal : Mémoire d'Encrier, 2011.

FONTAINE, Naomi. "Puamun, Le Rêve ». Littoral, n 10, printemps 2015, "L'Écriture innue", Pierre ROUXEL (dir.), p. 171.

FONTAINE, Naomi. " Je viens de là-bas ». In : BOUCHARD SERGE, DESY JEAN (dir.), Objectif Nord : Le Québec au-delà du 49, Montréal, Éditions Sylvain Harvey, 2013, p 44-47.

FONTAINE, Naomi. « Tshitissinat, notre territoire. Ce que tu dois savoir, Julie », texte inédit.

FONTAINE, Natasha Kanapé. Nanimissuat. Île-tonnerre. Montréal : Mémoire d'encrier, 2018.

FONTAINE, Natasha Kanapé. Bleuets et abricots. Montréal : Mémoire d'encrier, 2016. 
FONTAINE, Natasha Kanapé. Kuei, je te salue. Conversation sur le racisme. Montréal : Ecosociété, 2016.

FONTAINE, Natasha Kanapé. N'entre pas dans mon âme avec tes chaussures. Montréal : Mémoire d'encrier, 2012.

FONTAINE, Natasha Kanapé. Manifeste Assi. Montréal : Mémoire d'encrier, 2014.

GIL, Marie-Andrée. Béante. Chicoutimi : Editions La Peuplade, 2012.

GIL, Marie-Andrée. Frayer. Chicoutimi : Editions La Peuplade, 2015.

GRAÚNA, Graça. Flor da mata. Belo Horizonte: Penninha Edições, 2014.

GRAÚNA, Graça. Tear da palavra. Belo Horizonte: S.n., 2007.

GRAUNA, Graça. Tessituras da Terra. Belo Horizonte : Edições M.E, 2001.

GRAUNA, Graça. Canto Mestizo. Rio de Janeiro : Ed. Blocos, 1999.

KAMBEBA, Márcia Wayna. Ay Kakyri Tama. Eu moro na cidade. Manaus: Grafisa Gráfica e Editora, 2013.

KAPESH, An Antane. Je suis une Maudite sauvagesse. Ottawa : Editions Lémeac, 1976.

LEETRA INDÍGENA (éditée par MARTINS, Maria Sílvia Cintra). v. 2, n. 2, 2013. São Carlos: SP: Universidade Federal de São Carlos, Laboratório de Linguagens LEETRA.

LEETRA INDÍGENA (éditée par MARTINS, Maria Sílvia Cintra). "Parte 1 Escritas de mulheres ». v. 4, n. 1, 2014. São Carlos : SP : Universidade Federal de São Carlos, Laboratório de Linguagens LEETRA.

MACIEL, Márcia Nunes. "A história que ouvi da minha avó e o que aprendi com elas ». Rev. LEETRA, São Carlos-SP n. 4 v.1 2014, p. 10-16.

MESTOKOSHO, Rita. Née de la pluie et de la terre. Strasbourg : Editions Bruno Doucet, 2014.

MESTOKOSHO, Rita et DESY, Jean. Uashtessiu. Lumière d'automne. Montréal : Mémoire d'encrier, 2010.

POTIGUARA, Eliane. Metade cara, metade máscara. São Paulo: Global, 2004.

POTIGUARA, Eliane. A terra é a mãe do índio. Rio de Janeiro: Grumin, 1989. 
LA PLACE DES ÉCRIVAINES AUTOCHTONES DANS LA LITTÉRATURE MONDIALE: LE CAS DU BRÉSIL ET DU QUÉBEC

POTIGUARA, Eliane. Akajutibiró: terra do índio Potiguara. Unesco, 1994.

\section{Bibliographie générale}

ALMEIDA, Maria Inês de. Desocidentada. Experiência literária em terra indígena. Belo Horizonte: Editora UFMG, 2009.

ASSMANN, Jan. La Mémoire culturelle. Paris, Aubier, 2010.

ASSMANN, Aleida. Espaços da recordação. Formas e transformações da memória cultural. Campinas/SP : Editora da Unicamp, 2011.

AUDET, René. "L'autorité de la fiction dans des œuvres polytextuelles ». In : BOUJU, Emmanuel, L'autorité en littérature. Rennes : PUR, 2016, p. 133-139.

AUGE, Marc. Non-lieux : introduction à une anthropologie de la surmodernité. Paris : Seuil, 1992.

BELLEAU, Jean Philippe. Le mouvement indien au Brésil. Du village aux organisations. Rennes : PUR, 2014.

BERND, Zilá. Por uma estética dos vestígios memoriais. Belo Horizonte : Fino Traço, 2013.

BERND, Zilá (org.). Glossaire des mobilités culturelles. Bruxelles : P.I.E. Peter Lang, 2014.

BESSE, Jean-Marc. Le goût du monde. Exercices du paysage. Arles : Actes Sud/ENSP, 2009.

BIRRAUX, Pierrette. "Des Innus du Québec aux Nations Unies, en passant par les Yanomami du Brésil : un parcours (accidenté) de collaboration.», ["From the Innu of Quebec to the United Nations, via the Yanomami of Brazil: an eventful journey of collaboration", translation CHAUVET Laurent]. Justice spatiale spatial justice, $\mathrm{n}^{\circ} 11$ mars 2017 march 2017, http://www.jssj. org

BON, François. " Naomi Fontaine ", Le Tiers Livre, 22/05/2011, http://www.tierslivre.net/spip/spip.php?article2581

BOUCHARD, Gérard. Genèse des nations et culture du Nouveau Monde. Essai d'histoire comparée. Montréal : Boréal, 2000.

BOUCHARD, Gérard. L'Interculturalisme : un point de vue québécois. Montréal : Boréal, 2012. 
BOUCHARD, Gérard, ANDRES, Bernard. Mythes et sociétés des Amériques. Montréal : Éditions Québec Amérique, 2007.

BOUCHARD, Joë, CHARTIER, Daniel, NADEAU, Amélie (dir.). Problématiques de l'imaginaire du Nord. Montréal : UQAM/Figura, 2004.

BOUDREAU, Diane. Histoire de la littérature amérindienne au Québec. Montréal : Éditions de l'Hexagone, 1993.

CHARTIER, Daniel. " Définir des Modernités hybrides : entre société, patrimoine, savoir, pouvoirs contemporains et culture autochtones ». Globe. Revue internationale d'études québécoises, vol. 8, $\mathrm{n}^{\circ}$ 1, 2005, "Modernités amérindiennes et inuites", CHARTIER Daniel (dir.), p. 11-16.

CINTA LARGA, Pichuvy. Org. QUEIROZ, Ana Leonel de, WALTY, Ivete Lara Camargos, LEONEL, Leda Lima. Mantére ma Kwe tïnhim - Histórias de maloca antigamente. Belo Horizonte: SEGRAC-CIMI, 1988. Accessible sur le site

emad.fflch.usp.br/sites/lemad.fflch.usp.br/files/HISTÓRIASDEMALOCA ANTIGAMENTE.pdf

CRONON, William. Nature et récits. Essais d'histoire environnementale, traduit de l'américain par Mathias Lefèvre, Rhône-Alpes, Editions Dehors, 2016.

DANTAS, Beatriz G.; SAMPAIO, José Augusto L ; CARVALHO, Maria Rosário G. Os povos indígenas no Nordeste brasileiro. Um esboço histórico. In: CUNHA, Manuela Carneiro de. História dos índios no Brasil. São Paulo: Companhia das Letras, 2008, p. 431-456.

FIGUEIREDO, Eurídice. Políticas e poéticas da memória: gênero e etnicidade (Conceição Evaristo e Eliane Potiguara). In: Mulheres ao espelho: autobiografia, ficção, autoficção. Rio de Janeiro: EDUERJ, 2013, p. 149-167.

FONTAINE, Naomi. « Naomi Fontaine : Bons Baisers de la réserve », interview à Chantal Guy, La Presse-Canada, 13/05/2011,

http://www.lapresse.ca/arts/livres/entrevues/201105/13/01-4399063-naomifontaine-bons-baisers-de-la-reserve.php[consulté le 05/02/2016].

FOUILLEUL, Anaís. Les voix des femmes amérindiennes dans les littératures des Amériques. Mémoire soutenu le 20 / 09 / 2016, sous la direction de Rita Olivieri-Godet, dans le cadre du Master “Les Amériques” de Université Rennes 2. Accessible sur le site http://www.mulherespaz.org.br/acoes-das-mulheresda-paz/evento-jovens-na-campanha-nacional-mulheres-pela-paz/ DURAND, 
Monique. «Venir À L'Écriture ». Littoral, n 10, printemps 2015, “L'Écriture innue", ROUXEL, Pierre (dir.), p. 141-145.

GAGNE, Natacha et JEROME, Laurent (sous la direction de). Jeunesses autochtones. Affirmation, innovation et résistance dans les mondes contemporains. Rennes : PUR, 2009.

GATTI, Maurizio. Etre Écrivain amérindien au Québec : indianité et création littéraire. Montréal : Hurtubise / Cahiers du Québec, 2006.

GATTI, Maurizio. Littérature amérindienne du Québec. Ecrits de langue française. Montréal : Editions Hurtubise, 2004.

GEHLEN, Rejane Seitenfuss. "Identidade de Eliane: a face Potiguara, a máscara indígena e o eco de vozes silenciadas." In: Boitatá - Revista do GT de Literatura Oral e Popular da ANPOLL. Londrina, n. 12, p. 81-103, jul-dez 2011.

GRANJEAN, Pernette (sous la direction de). Construction identitaire et espace. Paris: L'Harmattan, 2009.

GRAÚNA, Graça. Contrapontos da literatura indígena contemporânea brasileira. Belo Horizonte: Maza Edições, 2013.

HAREL, Simon. Place aux littératures autochtones. Montréal : Mémoire d'Encrier, 2017.

HAREL, Simon. Les passages obligés de l'écriture migrante. Montréal : XYZ, 2005.

HAREL, Simon. Braconnages identitaires. Un Québec palimpseste. Montréal : VLB, 2006.

HAREL, Simon. Espaces en perdition. Tome I. Les lieux précaires de la vie quotidienne. Québec: PUL, 2007.

HAREL, Simon. Espaces en perdition. Tome II. Humanités jetables. Québec: PUL, 2008.

JECUPÉ, Kaká Werá. A terra dos mil povos: uma história do Brasil contada por um Índio. São Paulo: Peirópolis, 1998.

IMBERT, Patrick, " Les trois $\mathrm{R}$ - ruptures, route et réussite - dans les Amériques : entre l'oubli et la promesse ». BOUCHARD, Gérard, ANDRES, Bernard. Mythes et sociétés des Amériques. Montréal : Editions Québec Amérique, 2007, p. 139-169.

IMBERT, Patrick. Le transculturel et les littératures des Amériques. Le Canada et les Amériques. Ottawa, U Ottawa, 2012. 
IMBERT, Patrick. " Francophonies minoritaires : de l'homogène enraciné à la transculturalité de la société des savoirs ».

Américanité, cultures francophones canadiennes et société des savoirs. Le Canada et les Amériques. Ottawa, U Ottawa, 2009, pp. 15-66.

LE TOURNEAU, François-Michel et VELUT, Sébastien. " Les politiques et enjeux environnementaux ». In : QUENANT, C., VELUT, S. (coord.) Les enjeux du développement en Amérique latine : Dynamiques socioéconomiques et politiques publiques, Agence Française de Développement (AFD) ; Institut des Amériques (IDA), 201, pp. 321-367.

LE TOURNEAU, François-Michel. " En marge ou à la marge ? ", Espace populations sociétés [En ligne], 2014/2-3 |2015, mis en ligne le 01 décembre 2014 [consulté le 17/06/2017], URL : http://eps.revues.org/5859 ; DOI : 10.4000/eps.5859 LETOURNEAU, Jean-François. Le territoire dans les veines. Montréal : Mémoire d'encrier, 2017.

MORENCY, Jean. Le Mythe américain dans les fictions d'Amérique. Montréal : Nuit Blanche Éditeur, 1994.

MORISSET, Jean. Les chiens s'entre-dévorent. Indiens, Blancs et Métis dans le Grand Nord canadien. Montréal : Mémoire d'encrier, 2009.

LIMA, Antônio Carlos de Souza, BARROSO, Maria Macedo (org.). Povos indígenas e universidade no Brasil: contextos e perspectivas, 2004-2008. Rio de Janeiro : E-papers, 2013.

OLIVIERI-GODET, Rita. Écrire l'espace de l'Amérique: représentations littéraires et voix de femmes amérindiennes. New York: Peter Lang, Collection Brazilian Studies, 2019.

OLIVIERI-GODET, Rita. L'altérité amérindienne dans la fiction contemporaine des Amériques. Brésil, Argentine, Québec. Québec : Presses de l’Université Laval, 2015.

OLIVIERI-GODET, Rita. "Figurations des espaces amérindiens dans les littératures du Brésil et du Québec », Interfaces Brasil/Canadá, Vol 15, n² 2, décembre 2015. Expériences et écritures de l'espace au Québec et au Brésil, OLIVIERI-GODET, Rita, SOARES DE SOUZA, Licia, THIERION Brigitte (dir.),

https://periodicos.ufpel.edu.br/ojs2/index.php/interfaces/article/view/7282 $\angle 5101$

OLIVIERI-GODET, Rita, "Voz feminina ameríndia e escrita do espaço», Interfaces Brasil/Canadá, Vol 16, $\mathrm{n}^{\circ} 3$, dezembro 2016, “ À procura de novos paradigmas: estudos indígenas no Canadá e nas Américas”, Eloína Prati dos 
Santos, Rubelise da Cunha (dir.) https://periodicos. ufpel.edu.br/ojs2/index.php/interfaces/article/view/9386 PAGEAUX, Daniel-Henri. Littératures et cultures en dialogue. Paris: L'Harmattan, 2007.

POTIGUARA, Eliane. Interview. Accessible sur le site www.mulherespaz.org.br/acoes-das-mulheres-da-paz/evento-jovensnacampanha-nacional-mulheres-pela-paz/

REBRA, Rede de Escritoras Brasileiras, http://rebra.org/escritora/escritora_ptbr.php?id=1035 [consulté le 28/02/2017].

RIBEIRO, Darcy. O povo brasileiro: a formação e o sentido do Brasil. [S.1.]: Companhia das Letras, 1995, p. 470. ISBN: 9788571644519.

SANTILLI, Márcio. Os brasileiros e os índios. São Paulo: Senac, 2000.

SANTOS, Eloína Prati dos (Org.). Perspectivas da literatura ameríndia no Brasil, Estados Unidos e Canadá. Feira de Santana: Universidade Estadual de Feira de Santana, 2003. Accessible sur le site http://nec. furg. br/index.php/acervo. html

SOUZA, Lynn Mario T. Menezes. "Que história é essa? A escrita indígena no Brasil." In : SANTOS, Eloína Prati dos (Org.). Perspectivas da literatura ameríndia no Brasil, Estados Unidos e Canadá. Feira de Santana: Universidade estadual de Feira de Santana, 2003. Accessible sur le site http://nec.furg.br/index.php/acervo. html

SANTOS, Gilton Mendes dos, AMOROSO, Marta (orgs.). Paisagens Ameríndias. Lugares, circuitos e modos de vida na Amazônia. São Paulo, Terceiro Nome, 2013.

SIBONY, Daniel. Entre-deux. L'origine en partage. Paris : Seuil, 1991.

SIMARD, Jean-Jacques. La réduction. L'Autochtone inventé et les Amérindiens d'aujourd'hui. Sillery (Québec), Éditions du Septentrion, 2003.

SIOUI, Georges E.. Histoire amérindienne de l'Amérique. Québec/Paris : Les Presses de l'Université Laval / L'Harmattan, 2005.

THERY, Hervé. Le Brésil pays émergé. Paris: Armand Colin, 2014.

WALTER, Roland. "Prefácio”. In: GRAÚNA, Graça. Contrapontos da literatura indígena contemporânea brasileira. Belo Horizonte: Maza Edições, 2013, p. 913. 
WALTY, Ivete Lara Camargos. Narrativa e imaginário social: uma leitura das histórias de maloca antigamente de Pichuvy Cinta Larga. São Paulo: USP, 1991, 237 p. (Tese de Doutorado).

WALTY, Ivete Lara Camargos. $O$ intelectual $e$ as minorias. Site: http://lasa.international.pitt.edu/Lasa20oo/Walty.pdf [consulté le 28/03/2016].

YAMÃ, Yaguarê. Literatura indígena: da oralidade ao papel. Correio Braziliense, 3, Brasília, 29 de fevereiro de 2016. Accessible sur le site: ggrauna.blogspot.com/ [consulté le 1/03/2016].

Recebido em 18 de junho de 2019.

Aceito em 30 de junho de 2019. 